\title{
App-based Quantification of Crystal Phases and Amorphous Content in ZIFs Biocomposites
}

\author{
Michael R. Hafner, ${ }^{a}$ Laura Villanova, ${ }^{b *}$ and Francesco Carraro ${ }^{a *}$
}

a. Institute of Physical and Theoretical Chemistry, Graz University of Technology, 8010 Graz, Austria. E-Mail: francesco.carraro@tugraz.at

b. Faculty of Technical Chemistry, Chemical and Process Engineering, Biotechnology, Graz University of Technology, 8010 Graz, Austria. E-Mail: laura.villanova@tugraz.at

The performance of Zeolitic Imidazolate Frameworks (ZIFs) as protective hosts for proteins in drug delivery or biocatalysis strongly depends on the type of crystalline phases used for the encapsulation of the biomacromolecule (biomacromolecule@ZIF). Therefore, quantifying the different crystal phases, and the amount of amorphous content of ZIFs is becoming increasingly important for a better understanding of the structure-property relationship. Typically, crystalline ZIF phases are qualitatively identified from diffraction patterns. However, accurate phase examinations are time-consuming and require specialized expertise. Here, we propose a calibration procedure (internal standard $\mathrm{ZrO}_{2}$ ) for the rapid and quantitative analysis of crystalline and amorphous ZIF phases from diffraction patterns. We integrated the procedure into a user-friendly web application, named ZIF Phase Analysis, which facilitates ZIF-based data analysis. As a result, it is now possible to quantify $i$ ) the relative amount of various common crystal phases (sodalite, diamondoid, ZIF- $\mathrm{CO}_{3}-1$, ZIF-EC-1, U12 and ZIF-L) in biomacromolecule@ZIF biocomposites based on $\mathrm{Zn}^{2+}$ and 2-methylimidazole ( $\mathrm{HmIM}$ ) and ii) the crystalline-to-amorphous ratio. This new analysis tool will advance the research on ZIF biocomposites for drug delivery and biocatalysis.

Metal-Organic Frameworks (MOFs) are a class of coordination polymers that are formed via the assembly of inorganic nodes (metal clusters or ions) and organic linkers. ${ }^{1,2}$ Recent progress on composites based on MOFs and biomacromolecules, namely MOF biocomposites, have been generating significant interest in the field of biotechnology and biomedicine. ${ }^{3-6}$ In particular, Zeolitic Imidazolate Frameworks (ZIFs) prepared from $\mathrm{Zn}^{2+}$ and 2-methylimidizaole $(\mathrm{Hmlm})$ are widely studied because their facile synthesis is compatible with several different classes of biomacromolecules (e.g. proteins, carbohydrates, lipids, nucleic acids, viruses, and cells)..$^{3-5,7-11}$ By mixing biomacromlecules to a an aqueous solution of $\mathrm{Zn}$ cations and $\mathrm{HmIM}$, it is possible to find conditions inducing the spontaneous formation of the ZIF material around biomacromolecules (i.e. biomimetic mineralization). ${ }^{3}$ Once the ZIF biocomposite (biomacromolecule@ZIF) is formed, the encapsulated biomacromolecule can be protected against harsh environments ${ }^{3,12}$, can be stored without the need of refrigeration for long periods ${ }^{5,9,12}$ and can be released in a controlled fashion by exposing the biocomposite to chelating agents (e.g. EDTA) ${ }^{13}$, acidic environments (e.g., $\left.\mathrm{pH} \leq 6.5\right)^{13,14}$ and buffer solutions (e.g., PBS) ${ }^{13-15}$.

During the preparation of biomacromolecule@ZIF systems, reaction parameters (metal to ligand ratio, concentration, stirring or static conditions) and post-synthetic treatments (washing with water or ethanol) strongly influence the crystal structure of the $\mathrm{Zn}(\mathrm{mIM})_{2}$-based framework. ${ }^{3,16,17}$ The type of crystalline phase strongly influences the physical-chemical properties of the final biocomposite. ${ }^{16-20}$ For example, the diamondoid (dia) ZIF-8 is a MOF non-porous to $\mathrm{N}_{2}$ with a Brunauer-Emmett-Teller (BET) surface area of 40 $\mathrm{m}^{2} / \mathrm{g}^{21}$ whereas the sodalite (sod) ZIF-8 possesses permanent microporosity and has a BET surface area of $1200 \mathrm{~m}^{2} / \mathrm{g} .{ }^{21}$ Consequently, the different ZIF structures react differently towards external stimuli like $\mathrm{pH}$ changes (e.g. the release time of Bovine Serum Albumin (BSA) from dia ZIF-8 is 5 times longer than from sod ZIF-8 at pH 5.5). ${ }^{16}$ More peculiar properties were shown by amorphous ZIFs biocomposites: in drug delivery, $\mathrm{pH}$ changes can induce the instantaneous ZIF degradation and release of the biotherapeutics; ${ }^{11}$ in 
biocatalysis, an enhancement of the substrates diffusion and high enzymatic activities in enzyme@ZIF-8 biocatalysts were measured ${ }^{17}$. We note that, the quantification of the amorphous content is a challenging step that could require the combination of different characterization techniques (e.g. X-ray diffraction (XRD), $\mathrm{N}_{2}$ physisorption, thermogravimetric analysis, Infrared spectroscopy (IR), X-Ray photoemission spectroscopy). ${ }^{22}$
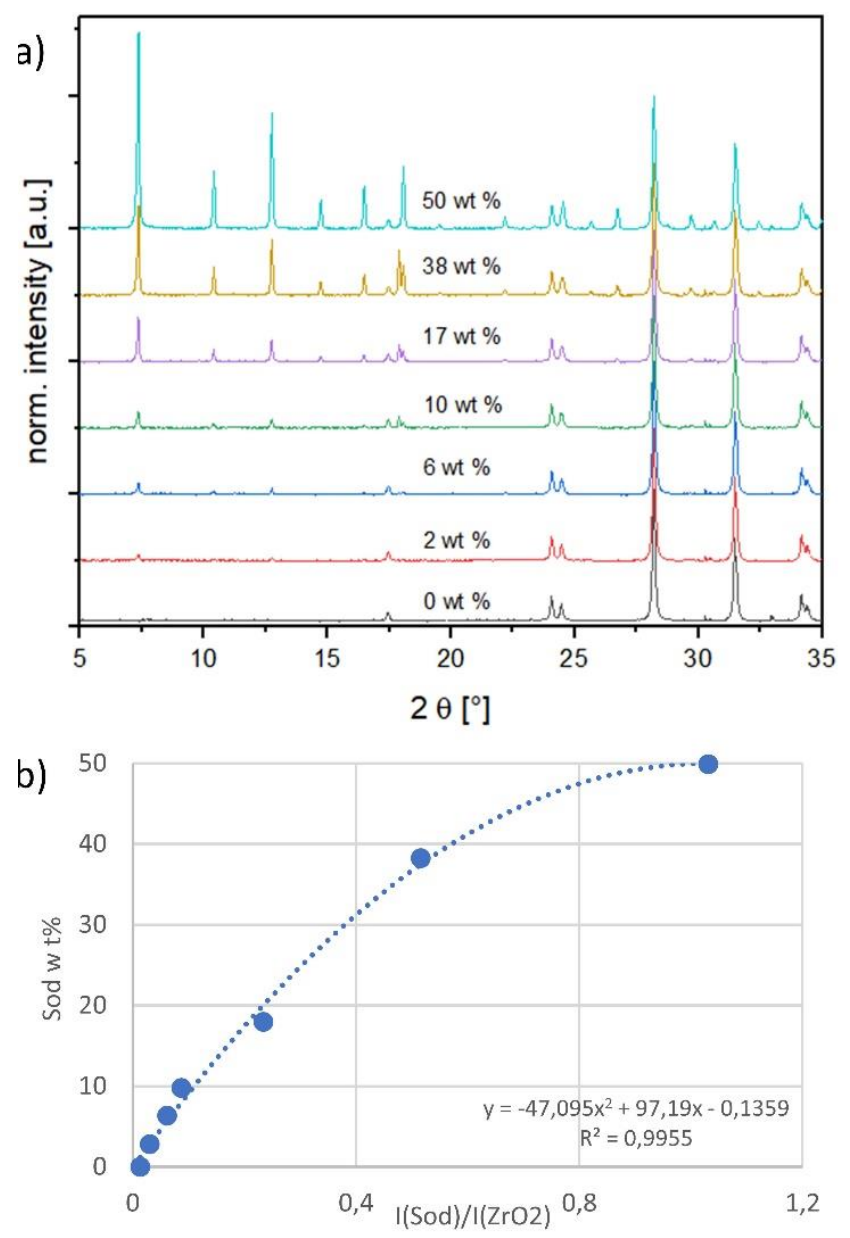

Figure 1: a) stacked PXRD patterns of $\mathrm{ZrO}_{2}$ with an increasing amount of sod, showing the amplifying peak at $7.36^{\circ}$; b) calibration curve of Sodalite. On the $y$-axis is the weight percentage (wt\%) of Sodalite in the mixture Sodalite/ $\mathrm{ZrO}_{2}$ and on the $\mathrm{x}$-axis is the ratio of the intensities of their respective quantification peaks

In general, a careful investigation of the obtained biocomposite typically shows a mixture of phases with a dominant component. As these phases have different functional properties an inaccurate phase identification results in erroneous assessments of their structure structure-property-function relationships. ${ }^{16}$ Thus, a tool that enables a rapid and accurate phase assessment in ZIFs will help the progress of biomacromolecule@ZIF composites in biomedicine and biocatalysis. ${ }^{16,22}$

Currently, a straightforward tool for the identification and quantification of both the crystalline phases and the amorphous fraction of ZIF biocomposites is missing. Furthermore, Powder X-Ray Diffraction (PXRD) analysis is among the most time-consuming steps in the development cycle of novel materials. ${ }^{23}$ To promote time effective and quantitative analysis of ZIFs biocomposites, we developed a web-based platform (ZIF Phase Analysis) that combines the customizable analytical capacities of the $\mathrm{R}$ environment ${ }^{24}$ and the intuitiveness of the user-friendly Shiny interface..$^{25}$ The platform is freely accessible via a dedicated website (https://rapps.tugraz.at/apps/porousbiotech/ZIFphaseanalysis/). It embeds a novel XRD calibration procedure that we developed to quantify the phases which are commonly found when synthesising ZIF 
biocomposites from $\mathrm{Zn}^{2+}$ and $\mathrm{HmIM}$ (i.e. sodalite and diamondoid ZIF-8, ZIF-CO $3-1$, ZIF-EC-1, U12, ZIF-L ${ }^{16,19,26-}$ ${ }^{28}$, here referred to as sod, dia, ZIF-C, ZIF-EC-1, U12 and ZIF-L) and quantify the amorphous content via the analysis of XRD patterns. This tool will facilitate the data analysis and accelerate the research in the emerging field of ZIF biocomposites, allowing researchers from different fields to precisely correlate the functional properties of the ZIF biocomposites (e.g. drug release profiles, biocatalytic activity) to the crystal phase(s) of the biocomposite.

\section{The calibration procedure}

We designed a calibration method with an internal standard $\left(\mathrm{ZrO}_{2}\right)$ for PXRD data analysis. $\mathrm{ZrO}_{2}$ was chosen as it is insoluble in water (thus it does not form a MOF with the 2-methylimidazole) and its PXRD diffraction peaks do not overlap ZIFs diffraction peaks. Firstly, by optimizing the synthesis conditions, we synthesised each phase - sod, dia, ZIF-C, ZIF-EC-1, U12 and ZIF-L - in a pure form excluding the formation of phase mixtures. These reaction conditions can be found in section S2.1. In Figure S1 the PXRD patterns of the different phases are compared to the available simulated patterns to ascertain the type and purity of the obtained phases. ${ }^{16,17,19,26}$ Secondly, increasing amounts of a phase are mixed with the internal standard in different weight fractions (ranging from 2 to $50 \mathrm{wt} \%$ ). The detailed procedure is given in section S3.1 and the resulting calibration curves are shown in Figure S3. Figure 1 depicts the calibration curve for sod. The relative sod weight percent (i.e. sod $w t \%)$ in the sod/ $\mathrm{ZrO}_{2}$ mixture is plotted in the $y$-axis, whereas the sod $/ \mathrm{ZrO}_{2}$ integrated intensities ratio (i.e. $\left.\mathrm{I}(\mathrm{sod}) / \mathrm{I}\left(\mathrm{ZrO}_{2}\right)\right)$ is plotted in the $\mathrm{x}$-axis. Each integrated intensity is calculated on the quantification peak (QP), namely the reflection in the diffractogram with the highest intensity. The sod QP is at $7.36^{\circ}$ and the $\mathrm{ZrO}_{2} \mathrm{QP}$ is at $28.20^{\circ}$ (20, $\mathrm{Cu} \mathrm{K} \alpha$ radiation). The QP for all phases are listed in Table S1. Thirdly, for each crystal phase we determined the ratio between the phase integrated intensity and weight \%. We then compared this intensity per weight ratio (phase/ $\mathrm{ZrO}_{2}$ ) to a common reference $\left(\mathrm{Al}_{2} \mathrm{O}_{3}\right.$, corundum) to experimentally derive an instrument-independent constant referred to as the reference intensity ratio (RIR, see Table S1 and section S5). ${ }^{29-31}$

\section{Crystal phases quantification based on experimental RIRs}

In this work, the calibration procedure was used to generate experimentally derived RIRs subsequently exploited for the investigation of ZIF phases (i.e. sod, dia, ZIF-C, ZIF-EC-1, U12 and ZIF-L). Such experimentally derived RIRs were not previously reported in the literature. Instead, the literature reports the ZIF crystal structure from which a RIR can be calculated. However, a calculated RIR does not account for the experimental conditions (e.g. absence of gas/liquid molecules in the pores) and could drastically differ from an experimentally derived RIR. In our study, the material is dried at room pressure and temperature (SI for details). Consequently, the calculated RIR differs from the experimentally derived RIR. This difference is more prominent for the porous sodalite (calculated RIR is $10.67^{32}$, experimentally found RIR is 4.5 ) and less prominent for the dense phases (e.g. dia: calculated RIR is $1.614^{33}$, experimental RIR is 2.6; ZIF-EC-1: calculated RIR is 1.403, experimental RIR is 2.5 ). For $\mathbf{U 1 2}$, the experimental RIR is 1.7 (since the crystal structure of $\boldsymbol{U 1 2}$ is not yet reported, there is not a calculated RIR available). In Table S1 the calculated and experimental RIRs of the ZIF phases are listed.

We used the experimentally derived RIRs to enhance the accuracy of a web application that was suitable for the identification of the main crystalline phase by using calculated or estimated RIRs. Additionally, we revisited the phase selection criteria (section S6), which now account for additional diffraction peaks and new ZIF phases. These changes resulted in an improved identification and quantification of crystalline phases, from traditional (sod ZIF-8) to recently discovered phases (ZIF-EC-1). 


\section{Amorphous content quantification (bi-phasic system)}

To quantify the amorphous content mixed to a pure ZIF phase (e.g. amorphous content in a sample that shows only sod diffraction peaks) we estimated the phase wt\% of the pure ZIF phase by using XRD data and its specific calibration curve (SI S3.3). Then, we compared this value with the expected phase wt\% (i.e. considering the ZIF $100 \%$ pure and crystalline). If these two values differ, we hypothesise that part of the material does not contribute to the intensities of the reflection peaks and postulate that the sample is a biphasic mixture where a certain proportion of the material is amorphous (e.g. amorphous/pure sod). ${ }^{31,34}$

To verify this hypothesis, we prepared selected protein@ZIF biocomposites that are already reported in the literature. ${ }^{16} \mathrm{BSA}$ is used as model protein and it is embedded in a ZIF matrix consisting of the sod, dia, ZIF-C and U12. The synthesis protocols are listed in Table S2.

With FT-IR spectroscopy we verified the formation of the BSA@ZIF composite materials (Figure S4). ${ }^{16}$

For structural analysis of the BSA@ZIFs composites, we measured with PXRD 1:1 weight mixtures of BSA@ZIFs composites and $\mathrm{ZrO}_{2}$ internal standard (prepared as in the calibration series, section S3.1). Figure 2 shows the diffractograms of the biocomposites. Depending on the reaction conditions (i.e. metal:ligand:BSA ratio) and the washing procedure (i.e. water or ethanol), different phases like sod (peaks at $7.36^{\circ}(110), 10.45^{\circ}(200)$ and $12.75^{\circ}(211)$ ), dia (peaks at $12.5^{\circ}(002), 13.05^{\circ}(011)$ and $13.75^{\circ}(20-2)$ ), ZIF-C (peak at $11.05^{\circ}(110)$ ) and $\mathbf{U 1 2}$ (peaks at $12.18^{\circ}$ and $18.43^{\circ}$ ) can be obtained. ${ }^{16}$

However, if the integrated intensity of the QP of the identified ZIF phase is inserted in the calibration curve, the calculated phase wt\% is lower than expected. This confirms the hypothesis that only a part of the weighed material contributes to diffraction and thus a certain amount is amorphous. In biocomposites, the formation of an amorphous content seems to be facilitated by the heterogeneous nucleation from the protein., $3,35-37$ The amount of encapsulated protein can be calculated via the analysis of the protein encapsulation efficiency (EE\% determined via Bradford assay, details are listed in section S4.3). ${ }^{16} \mathrm{EE} \%$ and measured gravimetric yield (Figure S5 and Figure S6, respectively) were used to calculate the weight of the encapsulated protein in the investigated sample. By subtracting the weight of the protein from the weight of the total biocomposite material, we determine the weight of the ZIF component for each sample. This theoretical ZIF wt\% is then compared to the wt\% calculated using the web app. The results of this comparison are shown in Figure 3 . For the analysed samples, the amorphous content ranges from $10 \%$ (sample 3) up to $30 \%$ (sample 2). Consequently, the amorphous phase is a substantial amount of the ZIF biocomposite: this should not be overlooked as it can alter the expected properties of the material. ${ }^{16,22,38,39}$
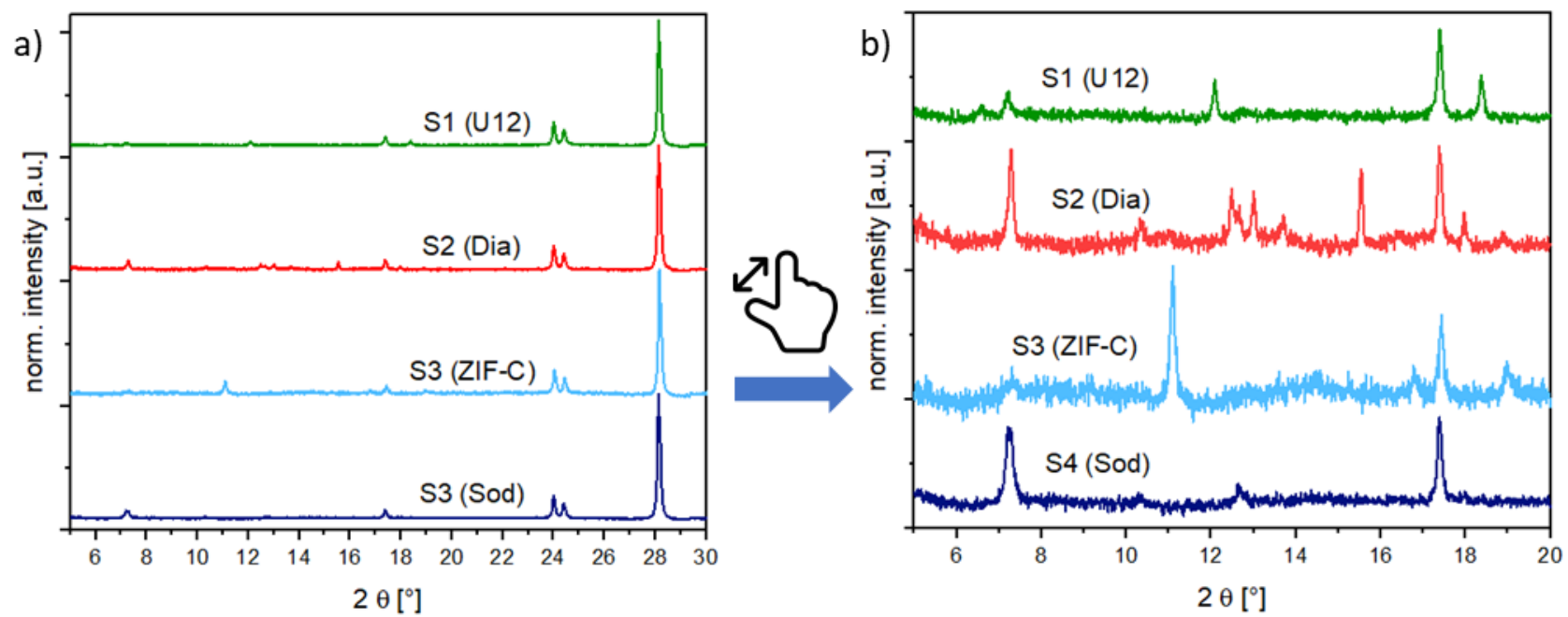

Figure 2: PXRD patterns of the BSA@ZIF biocomposite Samples (S1-S4) and their corresponding phases, $:$ Sodalite, 9 : Diamondoid, $:$ : U12, $:$ : ZIF-C; a) with 50 wt\% internal standard $\mathrm{ZrO}_{2} ;$ b) with $50 \mathrm{wt} \%$ internal standard $\mathrm{ZrO}_{2}$, zoomed into the region of the reflection signals of the ZIF phases. 


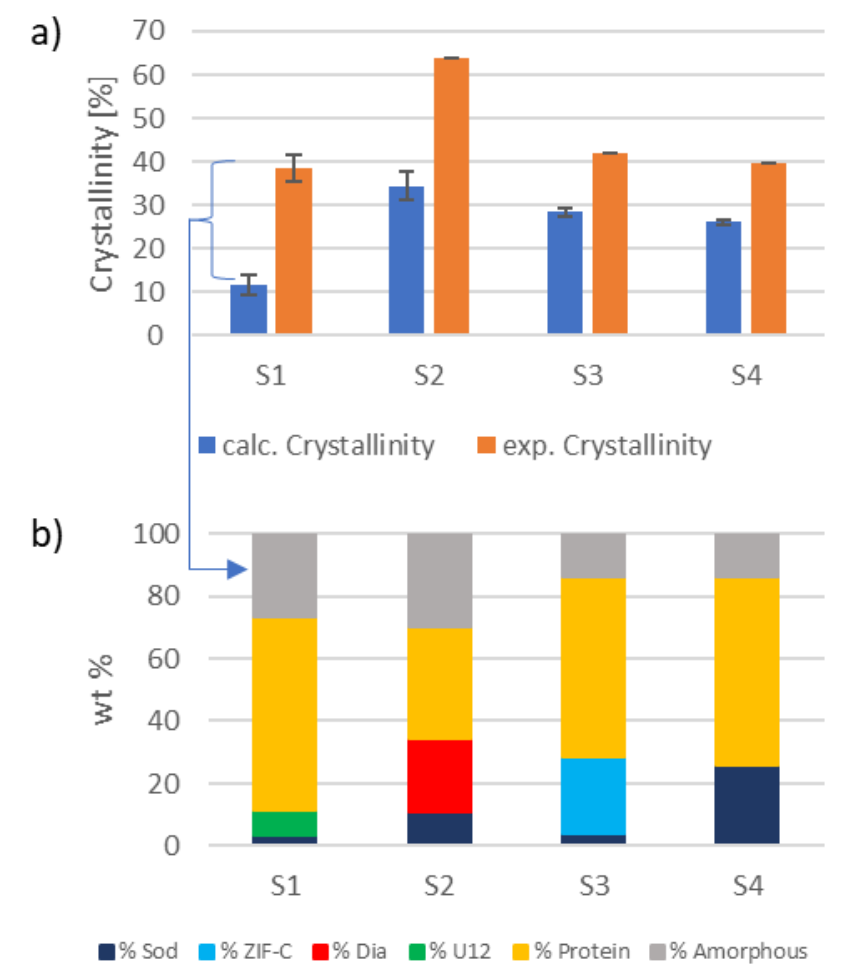

Figure 3: a) comparison of the calculated crystallinity, obtained through the calibration method with the experimentally found "crystallinity" (amount of ZIF) for the ZIF biocomposite samples (S1-S4); b) precise phase analysis of the ZIF biocomposite samples

(S1-S4) made by the calibration method.

Additionally, this procedure enables the precise evaluation of phase transitions during the processing of the same sample. For example, protein@ZIF-C biocomposites are often synthesized in water; then, when washed with ethanol, a phase transition from ZIF-C to sod can be observed. ${ }^{16}$ Here, we examined the conversion of BSA@ZIF-C (sample 3) to BSA@sod (sample 4) via ethanol washing. The quantification of the amorphous content in the two samples ( $15 \%$ in both cases) proves that the proportion of crystalline MOF is preserved during the transition from ZIF-C to sod. In fact, the ZIF- $\boldsymbol{C}$ to sod transition does not involve a sensitive loss of crystalline material (S3: ZIF-C wt\%= 24\%, sod $w t \%=2 \%$; S4: sod $w t \%=24 \%$ ) and supports the hypothesis that ZIF-C can be fully converted to sod.

\section{Web App implementation}

The ZIF Phase Analysis app consists of three sections that are shown in Figure 4. The Data Upload section is used to import the diffraction data (see video $\mathrm{SI}$ ). In this section it is possible to select multiple data file options such as the number of rows to skip and the column/decimal separators. Once the data are uploaded, it is possible to see the first few rows of the data file and the diffractogram.

The Multi-phase analysis section is used to analyse samples prepared without the $\mathrm{ZrO}_{2}$ internal standard. In this section it is possible to select the data analysis range by moving the extremes of the slider input to the desired $2 \theta$ values. We recommend using the $6^{\circ}-26^{\circ}$ range as it includes all the reference peaks of the detectable crystal phases. The entire $6^{\circ}-39^{\circ}$ range should be used if $\mathrm{ZrO}_{2}$ and/or $\mathrm{ZnO}$ are of interest as well. The analysis returns the identified phases and their relative proportions in the sample, along with the identified quantification peaks.

The Single-phase analysis section is used to analyse samples prepared with the $\mathrm{ZrO}_{2}$ internal standard. The user is required to input the phase wt $\%$ of the studied mixture (e.g. phase $w t \%=3 \%$ and $\mathrm{ZrO}_{2} \mathrm{wt} \%=97 \%$ ) and the crystal phase to be investigated. The $2 \theta$ values are automatically selected based on the crystal phase selected for analysis. The analysis returns the relative proportion of crystalline and amorphous material in the sample, as well as the identified quantification peaks for both crystal phase and internal standard. 


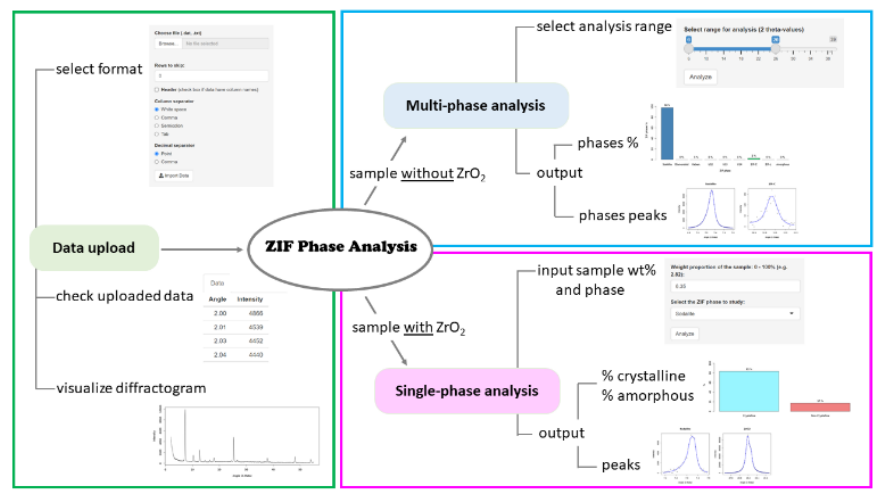

Figure 4: Workflow of the ZIF Phase Analysis app.

\section{Conclusion}

We built XRD calibration curves and experimentally determined the reference intensity ratio, RIR, of ZIF phases (sod, dia, ZIF-C, ZIF-L, U12 and ZIF-EC-1) that are commonly obtained when preparing ZIF biocomposites. The XRD calibration curves and experimental RIRs were obtained by mixing the different $\mathrm{Zn}(\mathrm{mIM}) 2$-based $\mathrm{ZIF}$ phases with an internal standard $\left(\mathrm{ZrO}_{2}\right)$ in different wt\%. The measured experimental RIRs were then used for the crystal phase identification and multiphase analyses. The calibration curves were employed to quantify the amorphous content in protein@ZIF biocomposite samples. For an accurate quantification, an internal standard $\left(\mathrm{ZrO}_{2}\right)$ was used and the related diffraction patterns analysed by a customized web application (ZIF phase analysis). By examining different protein@ZIF samples with our ZIF phase analysis app we estimated an amorphous content up to $30 \%$. Thus $1 / 3$ of the entire amount could significantly contribute to essential functional properties for biocatalysis or drug delivery (enzymatic activity and release profile respectively). Here we propose a procedure that requires the preparation of protein@ZIF samples and related standards (controlled mixtures of phases with a $\mathrm{ZrO}_{2}$ internal standard). The XRD patterns can then be uploaded in our ZIF phase analysis app. The freely available ZIF phase analysis app will promptly analyse the data providing an accurate evaluation of the different ZIF phase components, including the amount of the amorphous phase. As protein@ZIF materials is a burgeoning research field approached by scientists with diverse expertise (e.g. biology, enzymology, biomedicine), this app will especially support researchers with limited knowledge in crystallography. Thus, the here reported procedure combine with ZIF phase analysis web application will progress research of ZIF biocomposites for multi-disciplinary applications including drug delivery or biocatalysis. ${ }^{16,22,39}$

\section{Acknowledgments}

We thank Prof. Paolo Falcaro for supporting this project. We acknowledge European Union's Horizon 2020 Programme (FP/2014-2020)/ERC Grant Agreement no. 771834 POPCRYSTAL and TU Graz for the Lead Project (LP-03).

\section{Author Contributions}

M.R. Hafner: design of methodology, experiment implementation, data collection and sorting, discussion of the results and writing of the manuscript; L. Villanova: programming, software development, design and testing of web app, design and launch of website, revision of manuscript; F. Carraro: conceptualisation, design of methodology, supervision of the project, discussion of the results and writing of the manuscript.

\section{Conflicts of interest}

There are no conflicts to declare 


\section{References}

1 H.-C. Zhou, J. R. Long and O. M. Yaghi, Chemical Reviews, 2012, 112, 673-674.

2 H. Furukawa, K. E. Cordova, M. O’Keeffe and O. M. Yaghi, Science, 2013, 341, 1230444-1230444.

3 K. Liang, R. Ricco, C. M. Doherty, M. J. Styles, S. Bell, N. Kirby, S. Mudie, D. Haylock, A. J. Hill, C. J. Doonan and P. Falcaro, Nature Communications, , DOI:10.1038/ncomms8240.

4 E. Astria, M. Thonhofer, R. Ricco, W. Liang, A. Chemelli, A. Tarzia, K. Alt, C. E. Hagemeyer, J. Rattenberger, H. Schroettner, T. Wrodnigg, H. Amenitsch, D. M. Huang, C. J. Doonan and P. Falcaro, Materials Horizons, 2019, 6, 969-977.

5 F. C. Herbert, S. S. Abeyrathna, N. S. Abeyrathna, Y. H. Wijesundara, O. R. Brohlin, F. Carraro, H. Amenitsch, P. Falcaro, M. A. Luzuriaga, A. Durand-Silva, S. D. Diwakara, R. A. Smaldone, G. Meloni and J. J. Gassensmith, Nat Commun, 2021, 12, 2202.

6 Q. Qiu, H. Chen, Y. Wang and Y. Ying, Coordination Chemistry Reviews, 2019, 387, 60-78.

7 A. Poddar, J. J. Conesa, K. Liang, S. Dhakal, P. Reineck, G. Bryant, E. Pereiro, R. Ricco, H. Amenitsch, C. Doonan, X. Mulet, C. M. Doherty, P. Falcaro and R. Shukla, Small, 2019, 15, 1902268.

8 Y. Zhang, F. Wang, E. Ju, Z. Liu, Z. Chen, J. Ren and X. Qu, Adv. Funct. Mater., 2016, 26, 6454-6461.

9 R. Riccò, W. Liang, S. Li, J. J. Gassensmith, F. Caruso, C. Doonan and P. Falcaro, ACS Nano, 2018, 12, 13-23.

10 S. Li, M. Dharmarwardana, R. P. Welch, C. E. Benjamin, A. M. Shamir, S. O. Nielsen and J. J. Gassensmith, ACS Appl. Mater. Interfaces, 2018, 10, 18161-18169.

11 A. Poddar, S. Pyreddy, F. Carraro, S. Dhakal, A. Rassell, M. R. Field, T. S. Reddy, P. Falcaro, C. M. Doherty and R. Shukla, Chem. Commun., 2020, 56, 15406-15409.

12 Y. Feng, H. Wang, S. Zhang, Y. Zhao, J. Gao, Y. Zheng, P. Zhao, Z. Zhang, M. J. Zaworotko, P. Cheng, S. Ma and Y. Chen, Adv. Mater., 2019, 31, 1805148.

13 M. A. Luzuriaga, C. E. Benjamin, M. W. Gaertner, H. Lee, F. C. Herbert, S. Mallick and J. J. Gassensmith, Supramolecular Chemistry, 2019, 0, 1-6.

14 M. de J. Velásquez-Hernández, R. Ricco, F. Carraro, F. T. Limpoco, M. Linares-Moreau, E. Leitner, H. Wiltsche, J. Rattenberger, H. Schröttner, P. Frühwirt, E. M. Stadler, G. Gescheidt, H. Amenitsch, C. J. Doonan and P. Falcaro, CrystEngComm, 2019, 21, 4538-4544.

15 N. K. Maddigan, O. M. Linder-Patton, P. Falcaro, C. J. Sumby, S. G. Bell and C. J. Doonan, ACS Appl. Mater. Interfaces, 2021, 13, 51867-51875.

16 F. Carraro, M. de J. Velásquez-Hernández, E. Astria, W. Liang, L. Twight, C. Parise, M. Ge, Z. Huang, R. Ricco, X. Zou, L. Villanova, C. O. Kappe, C. Doonan and P. Falcaro, Chem. Sci., 2020, 11, 3397-3404.

17 W. Liang, R. Ricco, N. K. Maddigan, R. P. Dickinson, H. Xu, Q. Li, C. J. Sumby, S. G. Bell, P. Falcaro and C. J. Doonan, Chemistry of Materials, 2018, 30, 1069-1077.

18 M. Jian, B. Liu, R. Liu, J. Qu, H. Wang and X. Zhang, RSC Adv., 2015, 5, 48433-48441.

19 Y. Lo, C. H. Lam, C.-W. Chang, A.-C. Yang and D.-Y. Kang, RSC Adv., 2016, 6, 89148-89156.

20 Z.-X. Low, J. Yao, Q. Liu, M. He, Z. Wang, A. K. Suresh, J. Bellare and H. Wang, Crystal Growth \& Design, 2014, 14, 6589-6598. 
21 P. F. Rosen, J. J. Calvin, M. S. Dickson, A. D. Katsenis, T. Friščić, A. Navrotsky, N. L. Ross, A. I. Kolesnikov and B. F. Woodfield, The Journal of Chemical Thermodynamics, 2019, 136, 160-169.

22 X. Wu, H. Yue, Y. Zhang, X. Gao, X. Li, L. Wang, Y. Cao, M. Hou, H. An, L. Zhang, S. Li, J. Ma, H. Lin, Y. Fu, H. Gu, W. Lou, W. Wei, R. N. Zare and J. Ge, Nat Commun, 2019, 10, 5165.

23 F. Oviedo, Z. Ren, S. Sun, C. Settens, Z. Liu, N. T. P. Hartono, S. Ramasamy, B. L. DeCost, S. I. P. Tian, G. Romano, A. Gilad Kusne and T. Buonassisi, npj Comput Mater, 2019, 5, 1-9.

$24 \mathrm{R}$ Core Team, $\mathrm{R}$ : A language and environment for statistical computing, R Foundation for Statistical Computing, Vienna, Austria, 2019.

25 W. Chang, J. Cheng, J. J. Allaire, Y. Xie and J. McPherson, shiny: Web Application Framework for R, 2020.

26 M. Ge, Y. Wang, F. Carraro, W. Liang, M. Roostaeinia, S. Siahrostami, D. M. Proserpio, C. Doonan, P. Falcaro, H. Zheng, X. Zou and Z. Huang, Angew. Chem. Int. Ed., 2021, 60, 11391-11397.

27 R. Chen, J. Yao, Q. Gu, S. Smeets, C. Baerlocher, H. Gu, D. Zhu, W. Morris, O. M. Yaghi and H. Wang, Chem. Commun., 2013, 49, 9500.

28 S. A. Basnayake, J. Su, X. Zou and K. J. Balkus, Inorg. Chem., 2015, 54, 1816-1821.

29 F. H. Chung, J App/ Crystallogr, 2018, 51, 789-795.

30 X. Zhou, D. Liu, H. Bu, L. Deng, H. Liu, P. Yuan, P. Du and H. Song, Solid Earth Sciences, 2018, 3, 16-

29.

31 C. R. Hubbard and R. L. Snyder, Powder Diffr., 1988, 3, 74-77.

32 O. Shekhah, R. Swaidan, Y. Belmabkhout, M. du Plessis, T. Jacobs, L. J. Barbour, I. Pinnau and M. Eddaoudi, Chem. Commun., 2014, 50, 2089.

33 Q. Shi, Z. Chen, Z. Song, J. Li and J. Dong, Angew. Chem. Int. Ed., 2011, 50, 672-675.

34 C. R. Hubbard, E. H. Evans and D. K. Smith, J Appl Crystallogr, 1976, 9, 169-174.

35 K. Liang, C. Carbonell, M. J. Styles, R. Ricco, J. Cui, J. J. Richardson, D. Maspoch, F. Caruso and P. Falcaro, Advanced Materials, 2015, 27, 7293-7298.

36 X. Lian, Y. Fang, E. Joseph, Q. Wang, J. Li, S. Banerjee, C. Lollar, X. Wang and H.-C. Zhou, Chem. Soc. Rev., 2017, 46, 3386-3401.

37 F. Carraro, J. Williams, M. Linares-Moreau, C. Parise, W. Liang, H. Amenitsch, C. Doonan, C. O. Kappe and P. Falcaro, Angewandte Chemie International Edition, 59, 8123-8127.

38 T. D. Bennett, A. L. Goodwin, M. T. Dove, D. A. Keen, M. G. Tucker, E. R. Barney, A. K. Soper, E. G. Bithell, J.-C. Tan and A. K. Cheetham, Phys. Rev. Lett., 2010, 104, 115503.

39 C. Liu, J. Wang, J. Wan, Y. Cheng, R. Huang, C. Zhang, W. Hu, G. Wei and C. Yu, Angew. Chem. Int. Ed., 2020, 59, 3630-3637. 